\title{
Cardiac Disorders Associated with Cannabis: A Review of Literature Deepti Bhandare ${ }^{1 *}$, Christa Finer ${ }^{2}$ \\ 'Department of Cardiology, Advent Health Sebring, Sebring, Florida ${ }^{2}$ Medical Student, 4th year, Lake Erie College of Osteopathic Medicine, Bradenton, Florida
}

Article Info

\section{Article Notes}

Received: March 23, 2020

Accepted: April 16, 2020

\section{${ }^{*}$ Correspondence:}

Dr. Deepti Bhandare, MD, Department of Cardiology, Advent Health Sebring, 4638 Sun N Lake Blvd, Sebring, Florida, 33872 USA; Telephone No: 863386005;

Email: deeptibhandare81@gmail.com,

deepti.bhandare@adventhealth.com.

(c) 2020 Bhandare D. This article is distributed under the terms of the Creative Commons Attribution 4.0 International License.

\section{Keywords:}

Cannabis; Marijuana

Endocannabinoid system

Myocardial infarction

Stroke

Heart failure

\section{Abstract}

The leisure intake of cannabis has shortly amplified in the past years corresponding with its decriminalization and legalization. The natural cannabis has been substituted by synthetic cannabinoids and cannabimimetic in several formulae, which are stronger. In spite of irresistible public insight into the safety of these substances, a growing quantity of grave cardiovascular adverse events are reported in sequential relation to recreational cannabis intake. A multifaceted interface between the active ingredients, the endocannabinoid system, and the autonomic nervous system is responsible in the pathophysiology. Tolerance to the properties of cannabis can develop with repetitive contact due to receptor desensitization. Effects of cannabis may be heightened or transformed by affiliated use of other illicit drugs or drug treatment indicated for the treatment of cardiovascular diseases. Nonetheless, the recent cannabis epidemic would significantly increase global burden of cardiovascular diseases

\section{Introduction}

Marijuana plant known as Cannabis sativa was cultivated in Central Asia, and later transported to other parts of the world. Cannabis is a naturally growing plant and can be cultivated inside using hydroponic systems and non-natural lighting ${ }^{1}$. The plant has been used as a medicinal herb or as a mood-altering substance ${ }^{2}$. An increase in the utilization of cannabis has been recently noted due to new rules that that have endorsed legalization and tolerated the possession, sales, and cultivation of the cannabis ${ }^{3}$. The estimated users of cannabis are over 183 million in 2014 $4^{4}$. Cannabis is the most frequently used psychoactive substance after alcohol and tobacco. The recreational cannabis "epidemic" is accompanied by an elevated number of case reports of grave cardiovascular complications. We plan to assess probable cardiovascular adverse events associated with the use of these substances. The probable pathophysiological mechanisms accountable for the most serious cardiovascular events in cannabis users are discussed.

The literature review was performed in searches of EMBASE, PUBMED and MEDLINE databases utilizing the phrases "cannabinoids", "marijuana", "cannabis", "THC", "cardiovascular disease" and "stroke". All referenced articles were thoroughly reviewed, the findings were carefully examined for content accuracy. The existing literature was characterized conferring to significance and the clearness of the presented data. Attention was paid to exclude studies with strategies that diverged from normal conducts that cannabis was used recreationally. 


\section{Types Of Cannabis}

Cannabis comes in natural and synthetic forms.

\section{Cannabis: Natural Forms}

Cannabis occurs in three naturally growing strains (ruderalis, sativa and indica). Breeding has formed "cross" strains of the plants. These strains diverge in the amount of the two best recognized active ingredients, namely D9tetrahydrocannabinol (THC) and cannabidiol (CBD). The properties of various cannabis strains may fluctuate based on the variable concentration of terpene resins $s^{5,6}$. The sativa strain contains the maximum level of THC, the psychoactive cannabinoid that produces the euphoric effect and has a lower content of CBD, the cannabinoid that may alleviate the ecstatic and effects of THC. The highest content of THC is found in the leaves and buds of the female plants. These are dried for smoking. Other preparations like edibles, oils, waxes, vapor, liquid incense are also manufactured from cannabis. Cannabis is used for refractory nausea and vomiting following chemotherapy and neuropathic pain due to advanced neurologic disorders and cancer ${ }^{2,7,8}$. In the United States of America, cannabis is listed as a Schedule I substance as per section 202 of the Controlled Substances Act of 1970 by the Drug Enforcement Administration. Schedule I drugs have heightened potential for abuse, no currently well-known medical use, and no recognized care for usage with medical guidance. Forty Two states and the District of Columbia have legalized medical uses of cannabis.

A significant deliberation in the use of cannabis has been the randomness of the dosage of the active constituents of the artificially prepared forms. Comparable quantities of dried leaves/flowers, waxes/oils, or ingestible systems of cannabis could comprise immensely dissimilar amounts of active mixtures. The method of intake can impact the absorption, bioavailability and blood levels of the active mixtures. The plasma THC concentration and medical effects were alike after smoking and intravenous injection whereas ingestion caused smaller, deferred and lower peak plasma THC concentration in a study of healthy volunteers ${ }^{9,10}$. The possible physiologic effects of THC are also affected by the concomitant use of alcohol intake, nicotine intake and other illicit drugs. It is very essential to regulate the shelf life, bioavailability, dose range of the active compounds comprised of natural derivatives of medical cannabis preparations. A metered dose oral spray [Nabiximols], which has both THC and CBD, has been utilized for management of urinary dysfunction, spasticity and pain, in multiple sclerosis ${ }^{7}$.

\section{Synthetic Cannabis: Cannabinoids Cannabimimetics}

Synthetic THC compounds, dronabinol and nabilone, are available in capsule forms for management of weight loss associated with cancer chemotherapy and acquired immunodeficiency syndrome along with symptoms of nausea and vomiting. Illicit production of synthetic cannabinoids and cannabimimetics is utilized for recreational use. Given the advanced strength, it is more appealing to the users, and traditional drug screening tests are unable to categorize them. These constituents include a family of more than 700 synthetic compounds manufactured with chemical alterations of THC to augment the affinity for cannabinoid receptors and intensify the length of action. Synthetic cannabinoids are squirted onto dried leaves and marketed under various names including Spice $^{11}$

\section{Effects Of Cannabinoids On The Cardiovascular System}

Cannabis acts through the interaction of THC with the endocannabinoid system, an endogenous signaling network involved in a wide range of processes including immunity, endothelial function, inflammation and metabolism ${ }^{12}$. Two G protein-coupled membrane cannabinoid receptors, CBR1 and CBR2 are recognized. CBR1 is widely present in the nervous systems and is the main target of THC. The cardiovascular effects of cannabinoids are due to stimulation of the sympathetic nervous system, and inhibition of the parasympathetic nervous system ${ }^{12}$. Smoking cannabis results in tachycardia ${ }^{13}$. Certain studies have shown that the pretreatment with beta blockers like propranolol; atropine, and medications like rimonabant (reverse agonist of CBR1) prevent the CBR1-mediated modulation of the autonomic nervous system ${ }^{14-17}$. Cannabis also causes elevation of blood pressure ${ }^{18,19}$ and atrial fibrillation $^{20,21}$.

Smoking cannabis can decrease the oxygen transport to the heart and other vital organs by an elevation in blood carboxyhemoglobin levels and impaired myocardial oxygen demand to supply ratio ${ }^{22,23}$.

Smoking cannabis results in an instant elevation in heart rate, followed by a extensive rise in serum norepinephrine level. Acute exposure to cannabis causes elevation in systolic blood pressure and can induce various arrythmias like atrial fibrillation ${ }^{23}$.

Cannabis causes vasodilation by activating the transient receptor potential ankyrin type-1 (TRPA1) ion channels which are located on the perivascular sensory neurons, but the arteriolar vasodilation is not general to all vascular beds. Vasoconstriction is observed in the coronary, cerebral and peripheral arterial systems ${ }^{24,25}$.

\section{and Cardiovascular Complications Of Cannabis}

Cannabis users with cardiovascular emergencies are young and frequently have no other risk factors for 
cardiovascular disease. The incidence of cardiovascular complication rate has increased $1.1 \%$ in 2006 to $3.6 \%$ in 2010. The mortality rate from the cardiovascular complications was $25 \%{ }^{26}$. Rising fears have led researchers to call for a registry in the United States similar to the efforts of the French Addictovigilance Network ${ }^{27,28}$. Cardiovascular complications of cannabis use include acute myocardial infarction, arrhythmias, stroke, peripheral arteriopathy, stress cardiomyopathy (SC) and sudden death. The adverse events are usually noted in recreational users, but cases have been observed in those using the cannabis for medically approved reasons.

\section{Acute Myocardial Infarction [AMI]}

The incidence of AMI increases within an hour after exposure to cannabis $^{29}$. Frequently cannabis users presenting with AMI have been young men with no preexisting obstructive coronary artery disease $\mathrm{e}^{5,30}$. In general, AMI in cannabis users is linked with higher shortterm mortality as there is a delay in procuring medical care in young with people with diminished judgement due to cannabis use $\mathrm{e}^{31,32}$.

The drug screen has often been positive for cannabis only, although routine urine toxicology may be negative in those using synthetic cannabinoids ${ }^{33-35}$. This can cause marked underestimation of the AMI in the young cannabis users. Marijuana caused increased myocardial oxygen demand, reduced oxygen supply, and acts as a pro-coagulant ${ }^{36}$. Microvascular coronary artery spasm without atherosclerotic coronary artery disease have been described.

\section{Left Ventricular Cardiomyopathy}

The endocannabinoid structure is thought to be responsible in myocardial stunning, takotsubo [stress] cardiomyopathy, sepsis and hemorrhagic shock ${ }^{36,37}$. Cardiomyopathy can be due to tachycardia or atrial fibrillation $^{39}$.

The catecholamine rush may be largely responsible for the association. The presence of pre-existing myocardial dysfunction can predispose to deterioration of left ventricular systolic function particularly with the use of potent synthetic cannabinoids ${ }^{38}$.

\section{Arrythmias and Sudden Cardiac Death}

Rhythm disturbances range from sinus tachycardia to ectopic atrial or ventricular rhythms, and atrial or ventricular fibrillation in cannabis users, the pathophysiology being a hyperadrenergic state ${ }^{40}$. Cardiac ischemia due to microvascular spasm of acute coronary syndrome can cause fatal ventricular arrhythmias. These can cause dizziness, syncope, cardiac arrest or sudden cardiac death. Increased incidence of atrial fibrillation in the younger (less than 50 years old) individuals and recurrence may occur upon repeated cannabis exposure ${ }^{41}$.

\section{Cerebrovascular syndrome}

Neurological indications were the most common reasons $(44 \%)$ for hospitalization of cannabis users in French healthcare facilities from 2004 to $2007^{5,42}$.

Patient admitted with stroke and substance abuse, cannabis was linked with ischemic but not hemorrhagic events, while amphetamines were related to predominantly hemorrhagic events ${ }^{43}$. Comparable outcomes were reported from the United States Nationwide Inpatient Sample, cannabis was an independent prognosticator of acute stroke and associated with a threefold increase in hospitalization with acute ischemic stroke ${ }^{44}$.

The ischemic strokes are frequently noted in the posterior cerebral circulation, the principal mechanism of stroke was noted to be reversible cerebrovascular spasm (RCVS). RCVS causes severe headache, photophobia, vomiting, blurred vision and a "string and beads" appearance of cerebral arteries on angiography ${ }^{45}$. Multifocal tightening of cerebral arteries in the posterior circulation was predictive of cannabis use $^{46}$. Reversibility of cerebral vasoconstriction was noted on angiography within days after cessation of cannabis use ${ }^{47}$.

\section{Vasculopathy}

Cannabis vasculopathy is clinically inseparable from thromboangiitis obliterans but tends to occur at an earlier age. The angiography shows segmental narrowing of distal arteries with minimal collateralization ${ }^{48,49}$.

Arteritis in the extremities can lead to tissue necrosis and gangrene. Other vascular problems of cannabis use have included migratory thrombophlebitis, renal artery dissection and central retinal vein occlusion ${ }^{40,50}$.

\section{Conclusions}

Cannabis use has increased in epidemic proportions; extremely strong and unregulated synthetic cannabinoids and cannabimimetics have made the arena difficult. Cardiovascular symptoms have been occurring at a startling rate in users of cannabis and its related chemicals. Grave illness has occurred in young adults who are profound users of these compounds due to the wrong belief of them being safer, and swift decriminalization and legalization processes in the various states. Studies are greatly required in this expanse.

The current data should make physicians and legislators aware across the globe of the potentially hazardous effects of the unregulated use of cannabis. 


\section{References}

1. United Nations Office on Drugs and Crime (UNODC). World drug report 2016. New York: United Nations Publication; 2016 [Accessed 26 July 2017] p. 174. http://www.unodc.org/doc/wdr2016/WORLD_ DRUG_REPORT_2016_web.pdf.

2. Iversen LL. The science of marijuana. Br J Clin Pharmacol. 2009; 67: 268.

3. Center for Behavioral Health Statistics and Quality. 2015 National survey on drug use and health: detailed tables prevalence estimates. $2016 . \quad$ https://www.samhsa.gov/data/sites/default/files/ NSDUHDetTabs-2016/NSDUH-DetTabs-2016.pdf.

4. Substance Abuse and Mental Health Services Administration. Results from the 2011 national survey on drug use and health: summary of national findings, NSDUH series $\mathrm{H}-44$, HHS publication no. (SMA) 12-4713. Rockville, Maryland: Substance Abuse and Mental Health Services Administrations, 2012.

5. Jouanjus E, Raymond V, Lapeyre-Mestre M, et al. What is the current knowledge about the cardiovascular risk for users of cannabis-based products? A systematic review. Curr Atheroscler Rep. 2017; 19: 26.

6. Booth JK, Page JE, Bohlmann J. Terpene synthases from Cannabis sativa. PLoS One. 2017; 12: e0173911.

7. Koppel BS, Brust JC, Fife T, et al. Efficacy and safety of medical marijuana in selected neurologic disorders. Report of the Guideline Development Subcommittee of the American Academy of Neurology. Neurology. 2014; 82: 1556-63.

8. Iversen L. Cannabis and the brain. Brain. 2003; 126: 1252-70.

9. Ohlsson A, Lindgren JE, Wahlen A, et al. Plasma delta-9 tetrahydrocannabinol concentrations and clinical effects after oral and intravenous administration and smoking. Clin Pharmacol Ther 1980; 28: 409-16.

10. Grotenhermen F. Pharmacokinetics and pharmacodynamics of cannabinoids. Clin Pharmacokinet. 2003; 42: 327-60.

11. Gurney SMR, Scott KS, Kacinko SL, et al. Pharmacology, toxicology, and adverse effects of synthetic cannabinoid drugs. Forensic Sci Rev. 2014; 26: 53-78.

12. Benowitz NL, Rosenberg J, Rogers W, et al. Cardiovascular effects of intravenous delta-9-tetrahydrocannabinol: autonomic nervous mechanisms. Clin Pharmacol Ther. 1979; 25: 440-6.

13. Gash A, Karliner JS, Janowsky D, et al. Effects of smoking marihuana on left ventricular performance and plasma norepinephrine studies in normal men. Ann Intern Med. 1978; 89: 448-52.

14. Kanakis C Jr, Pouget JM, Rosen KM. The effects of delta-9tetrahydrocannabinol (cannabis) on cardiac performance with and without beta blockade. Circulation. 1976; 53: 703-7.

15. Beaconsfield P, Ginsburg J, Rainsbury R. Marihuana smoking: cardiovascular effects in man and possible mechanisms. M Engl J Med. 1972; 287: 209-12.

16. Sulkowski A, Vachon L, Rich E. Propranolol effects on acute marihuana intoxication in man. Psychopharmacology. 1977; 52: 47-53.

17. Huestis MA, Boyd SJ, Heishman SJ, et al. Single and multiple doses of rimonabant antagonize acute effects of smoked cannabis in male cannabis users. Psychopharmacology. 2007; 194: 505-15.

18. Karschner EL, Darwin WD, McMahon RP, et al. Subjective and physiological effects after controlled Sativex and oral THC administration. Clin Pharmacol Ther. 2011; 89: 400-7.

19. Jicha CJ, Lofwall MR, Nuzzo PA, et al. Safety of oral dronabinol during opioid withdrawal in humans. Drug Alcohol Depend. 2015; 157: 17983.

20. Charbonney E, Sztajzel JM, Poletti PA, et al. Paroxysmal atrial fibrillation after recreational marijuana smoking: another "holiday heart"? Swiss Med Wkly. 2005; 135: 412-4.

21. Kosior DA, Filipiak KJ, Stolarz P, et al. Paroxysmal atrial fibrillation following marijuana intoxication: a two-case report of possible association. Int J Cardiol. 2001; 78: 183-4.

22. Wu TC, Tashkin DP, Djahed B, et al. Pulmonary hazards of smoking marijuana as compared with tobacco. N Engl J Med. 1988; 318: 34751.

23. Aronow WS, Cassidy J. Effect of marihuana and placebo-marihuana smoking on angina pectoris. N Engl J Med. 1974; 291: 65-7.

24. Jordt SE, Bautista DM, Chuang HH, et al. Mustard oils and cannabinoids excite sensory nerve fibres through the TRP channel ANKTM1. Nature. 2004; 427: 260-5.

25. Dines AM, Wood DM, Galicia M, et al. Presentations to the emergency department following cannabis use-a multi-centre case series from ten European countries. J Med Toxicol. 2015; 11(4): 415-21.

26. Jouanjus E, Lapeyre-Mestre M, Micallef J. Cannabis use: signal of increasing risk of serious cardiovascular disorders. J Am Heart Assoc. 2014; 3: e000638.

27. Rezkalla S, Kloner RA. Recreational marijuana use: is it safe for your patient? J Am Heart Assoc. 2014; 3: e000904.

28. Rezkalla S, Stankowski R, Kloner RA. Cardiovascular effects of marijuana. J Cardiovasc Pharmacol Ther. 2016; 21: 452-5.

29. Mittleman MA, Lewis RA, Maclure M, et al. Triggering myocardial infarction by marijuana. Circulation. 2001; 103: 2805-9.

30. Caldicott DG, Holmes J, Roberts-Thomson KC, et al. Keep off the grass: marijuana use and Cardiol Ther (2018) 7:45-59 55 acute cardiovascular events. Eur J Emerg Med. 2005; 12: 236-44.

31. Mukamal KJ, Maclure M, Muller JE, et al. An exploratory prospective study of marijuana uses and mortality following acute myocardial infarction. Am Heart J. 2008; 155: 465-70.

32. Hall W, Solowij N. Adverse effects of cannabis. Lancet. 1998; 352: 1611-6.

33. Mir A, Obafemi A, Young A, et al. Myocardial infarction associated with use of the synthetic cannabinoid K2. Pediatrics. 2011; 128: e1622-7.

34. Atik SU, Dedeoglu R, Varol F, e al. Cardiovascular side effects related with use of "bonzai": two case reports. Turk Arch Pediatr. 2015; 50: $61-4$

35. McKeever RG, Vearrier D, Jacobs D, et al. K2-not the spice of life; synthetic cannabinoids and ST elevation myocardial infarction: a case report. J Am Coll Med Toxicol. 2015; 11: 129-31.

36. Hodcroft CJ, Rossiter MC, Buch AN. Cannabis-associated myocardial infarction in a young man with normal coronary arteries. J Emerg Med. 2014; 47: 277-81.

37. Bonz A, Laser M, Ku"llmer S, et al. Cannabinoids acting on CB1 receptors decrease contractile performance in human atrial muscle. J Cardiovasc Pharmacol. 2003; 41: 657-64.

38. Mukhopadhyay P, Ba'tkai S, Rajesh M, et al. Pharmacological inhibition of CB1 cannabinoid receptor protects against doxorubicin-induced cardiotoxicity. J Am Coll Cardiol. 2007; 50: 528-36

39. Godlewski G, Malinowska B, Schlicker E. Presynaptic cannabinoid $\mathrm{CB}$ (1) receptors are involved in the inhibition of the neurogenic vasopressor response during septic shock in pithed rats. $\mathrm{Br} J$ Pharmacol. 2004; 142: 701-8.

40. Singh A. Saluja S, Kumar A. Cardiovascular Complications of Marijuana and Related Substances: A Review. Cardiol Ther. 2018; 7: 45-59.

41. Korantzopoulos P. Marijuana smoking is associated with atrial fibrillation. Am J Cardiol. 2014; 113: 1085-6. 
42. Jouanjus E, Leymarie F, Tubery $\mathrm{M}$, et al. Cannabisrelated hospitalizations: unexpected serious events identified through hospital databases. Br J Clin Pharmacol. 2011; 71: 758-65.

43. Westover AN, McBride S, Haley RW. Stroke in young adults who abuse amphetamines or cocaine: a population-based study of hospitalized patients. Arch Gen Psychiatry. 2007; 64: 495-502.

44. Rumalla K, Reddy AY, Mittal MK. Recreational marijuana use and acute ischemic stroke: a population- based analysis of hospitalized patients in the United States. J Neurol Sci. 2016; 364: 191-6.

45. Melki E, Denier C, The'audin-Saliou M, et al. External carotid artery branches involvement in reversible cerebral vasoconstriction syndrome. J Neurol Sci. 2012; 313: 46-7.

46. Ducros A, Boukobza M, Porcher $\mathrm{R}$, et al. The clinical and radiological spectrum of reversible cerebral vasoconstriction syndrome. A prospective series of 67 patients. Brain. 2007; 130: 3091-101.

47. Wolff V, Lauer V, Rouyer O, et al. Cannabis use, ischemic stroke, and multifocal intracranial vasoconstriction a prospective study in 48 consecutive young patients. Stroke. 2011; 42: 1778-80.

48. Noel B, Ruf I, Panizzon RG. Cannabis arteritis. J Am Acad Dermatol. 2008; 58(Suppl 1): S65-7.

49. Schneider F, Abdoucheli-Baudot N, Tassart M, et al. Cannabis and tobacco: cofactors favoring juvenile obliterative arteriopathy. J Mal Vasc. 2000; 25: 388-9.

50. Disdier P, Granel B, Serratrice J, et al. Cannabis arteritis revisited-ten new case reports. Angiology. 2001; 52: 1-5. 